\title{
The Role of Cyberspace in Learning the Persian Language
}

\author{
Somayeh Aghajani Kalkhoran \\ Hankuk University of Foreign Studies, Seoul, South Korea
}

\begin{abstract}
In today's modern world there is an important issue which is how media especially, the internet threatens our language. It is undoubtedly true that media directly or not, has a substantial influence on language. This paper focuses on how this menace, "Cyberspace", makes opportunities for languages to be learned in all over the world. It seems that although cyberspace and especially the internet have some unfavorable effects on languages, they offer a big help in several ways to language learners easily become familiar with all aspects of the new language. This paper tried to find opportunities and threats which the internet brings about to learning Persian language and how they affect learning this language as a new one. It seems that albeit there are many threats to Persian language and its learning on the internet such as existence of writing-speaking mixture of Persian language, decreasing the attention to resource books and so on, the opportunities which caused by the internet, such as avalable resource everywher for everyone in anytime, cheeper or free education and so on, outweigh the negative effects and it is generally accepted we cannot omit cyberspace especially the internet from people's lives. Therefore, experts have to use the advantages of the internet and technology to sustain and develop languages.
\end{abstract}

Keywords: cyberspace, internet, threats and opportunities, learning Persian language

\section{Introduction}

Language is commonly used as a tool for communication. Our thoughts also represent themselves in the form of words when they come into existence, and even in our minds, in order to decide or do any mental work, we must first put the mind contents in the form of language. As a result, the most important means of communication, science, advancement, and technology in each society is the language. Language in its cotemporary meaning, can be seen in several categories like written, oral, pictures, and symbols, each of which are proportionate to different languages and cultures and of course, are changed during time. The dimension which this article going to discuss is written form of language.

As time goes on, languages have been changed in all over the world while these changes are somehow slowly and undisguisable that we cannot feel it. David Crystal, professor of linguistics at the University of Bangor, believes that: "Language itself changes slowly but the internet has speeded up the process of those changes so you notice them more quickly" (Kleinman, 2010).

Insomuch, a great part of our written language nowadays is on computers, tablets, and other tools of cyberspace; our language is the result of interaction with technology. Due to this interaction, language is changing subtly every day. This alternation could be seen easily in speaking form of language, unlike writing form which is gentle. We are faced with new things since, we use, name, and call them in contrast; we put away

Somayeh Aghajani Kalkhoran, Ph.D., Assistant Professor, Persian department, Hankuk University of Foreign Studies, Seoul, South Korea. 
somethings because they are antiquated or we less use them or even call them in another form because of our fast-paced modern life that people do not have enough time to speak or write in long shape; the solution is short form.

We spend a huge part of our daily time online rather than watching TV or listening to the radio. Therefore, the internet has the most effect on our lives. In spite of the fact that most of the people all over the world have an ease of access to cyberspace (in its various forms) while Iranian people are deprived of them because of filtering of some social networks such as Facebook and Wechat, people use an extraordinarily wide variety of social networks.

What cyberspace exactly is? Like many words in the world, people have given definitions during years. The first one who used it was Gibson:

Visually, cyberspace is a three-dimensional representation of all the bases which look like geometric shapes. These bases are laid out in specific zones of the horizontal cyberspace grid. In actuality, cyberspace is just an image generated in your brain by your cyberspace deck. Without the deck, cyberspace would just be a complex set of information that human would never be able to understand. (Gibson, 1984, p. 4)

The form of using this communication tool in Iran has its own characteristics. No matter when, where, and how cyberspace is used, it causes effects. Bourdieu (1992) are of the belief that, complicated and inveterate statuses are forming values of society and temperaments, subconsciously.

He believes that social interactions, patterns of action and comprehension are built through various areas of daily life such as education, family life, spare time, and so on. Daily communication methods form these areas and online social networks can be signs and samples of new forms of mentioned communicative manner.

This perceptual frame is utilized to the study of language as a kind of wealth in generation and reconstruction of language (Bourdieu, 1992, pp. 43-65). As language important in everyday life and it is a communication tool, changing language causes changing culture eventually.

In this research, the potential, threats, and opportunities of the cyberspace, especially the internet are addressed to the Persian language and survey displays that what are the opportunities and threats of the internet for teaching and learning the Persian language and what are the possible solutions to control the powerful undesirable effects of networks on learning the Persian language.

\section{Persian Language}

One of the Indo-European languages in the Southwestern Iranian language branch is Persian

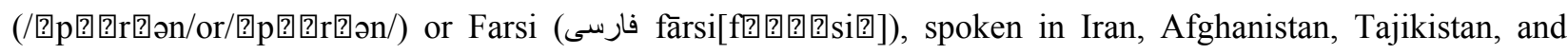
Uzbekistan. Persian is the official language of Iran and Tajikistan and is one of the official languages of Afghanistan (along with Pashto). The official language of India was also Farsi before colonization by England. Moreover, Farsi had been used in various areas that were considered as parts of the Greater Iran. This language is written in Persian alphabet which is a modified version of Arabic script.

Farsi has been a distinguished cultural language in different parts of South, Central, and Western Asia under the rule of various empires. Persian speakers population all over the world are about 110 million people. It has been the official language of Iran, Afghanistan, and Tajikistan for many years.

Many neighbor languages had been influenced by Farsi such as Turkic languages in different parts of Asia, Armenian, Georgian, Indo-Aryan languages, and especially Urdu. Although Persian borrowed plenty of words from Arabic after the Arab conquest of Iran, Farsi has had some effects on it. 
What is the role of Persian language and how is its presence on the internet? The majority of the population in Iran speak Persian and use cyberspace. Although this number increases,

only $0.8 \%$ of the content of cyberspace (even less than $1 \%$ ) is in Persian language. That is about $57 \%$ for English! This issue, in addition to the prevalence of a particular style of the Persian language in social networks among young generation, known as the Internet language, is full of spelling mistakes and false idioms have been a source of concern for the cultural authorities of the country. ("Cyberspace; A Tool of Threat or an Opportunity”, 1393)

No one can ignore the influence of the internet on languages, as Persian is one of them. These days we use the internet almost for everything; selling and buying food, fruits, jewelry, carpet, car, online tickets, watching movies and various matches, chat and communicate with people in all over the world and all that can be imagined. It seems that governmental and non-governmental organizations have been making efforts to "rescue the Persian language", but it is clear that these tries have not been sufficient and we need more and more collaborative endeavors from all institutions and individuals in Iranian community. Nowadays the cyberspace is not just an abstract concept but it has its reality:

With cyberspace the real world (let us grant some consensus here as to its physicality) does not become etherealized and thus, in the aggregate, less large or less real, nor does the "mental" world become concrete and thus, itself, less mental or spiritual. Rather, with cyberspace, is whole new space is opened up by the very complexity of life: a new niche for a realm that spreads between the two worlds. Cyberspace becomes another venue for conciseness itself and this emergence, proliferation, and complexification of conciseness must surely be its universe's projection. (Benedikt, 1991, p. 124)

The first step in reducing the internet challenges for the Persian language and its learning, is to recognize the threats and opportunities of cyberspace to learn this language.

There are opportunities and threats in using the internet not just for language but for all life dimensions.

As bandwidth burgeon and computing muscle continue to grow, cyberspace places present themselves in increasingly multisensory and engaging ways. They will look, sound and feel more realistic, they will enable richer self-representation of their users, they will respond to user actions in real time and in complex ways and they will be increasingly elaborate and artfully designed. We will not just look at them; we will feel present in them. (Mitchel, 1995, p. 114)

We share more personal information and also communicate with a wide different range of people. Our communication ways consequently become more informal and more open, and this causes transfusion into other areas of life and culture.

In this way, we utilize language through the internet to do all these activities. There is a rule for all languages which says all speakers of a language try to spend less energy to speak.

In addition to this tendency, the nature of the internet and greater, Cyberspace, intensifies changing languages (at least spoken form) to become easier.

Nowadays popular social networks prepare and represent their services in different languages which have more speakers all over the world whereas this does not include all of the languages even those which have millions of speakers. Advanced technology brings about the creation of new words or change in the use of existing words. For example, "wall", "wireless", "tablet", "tag", "follow", and "like"-although all of these words have their own normal meanings, recently they are utilized in a totally different situation. Internet and especially communicative sites and applications offer many spaces to establish a connection such as online-chat, blogs, forums, Twitter, instant messaging, etc., each of which have their own rules, limitations, and norms for language use. 
Some people claim that cyberspace has reduced the literacy specially among young generation and some disagree:

The well-known British linguist David Crystal largely dismisses the common view that online communication is illiterate and dumbed-down language. He agrees that much of it is non-standard, playful, highly deviant in bending the usual rules of language, tolerant of typographic and spelling errors, and full of new words. But he is fascinated by its variety and innovation and takes a very positive view, suggesting that "The phenomenon of Netspeak is going to change the way we think about language in a fundamental way, because it is a linguistic singularity-a genuine new medium". (Crystal, 2001, p. 238)

\section{Threats and Opportunities}

\section{Threats}

Not access to main sources. The prevalence of slangy writing can be confusing for new learners who do not have access to the main sources and it causes they consider casual writing as the original and correct form of the language.

Users who seek to learn Persian language through cyberspace and the internet, if they do not have access to the formal and standard texts of this language and just be satisfied with the internet, are likely to be faced with deformed, slang, informal spoken and abbreviated language. In spoken and colloquial languages, not only words are shortened, or not the formal one, but the grammar is also different and does not follow the formal rules.

The word-shifting and its repetition during time would change the original form of the words in undesirable way.

Internet users, for some reasons, limit the volume or amount of their internet consumption; they try to write down their content in a concise way, and express more information to their audience with less description. Therefore, they choose the abbreviation causes the creation of special acronyms. The original words are replaced with these modified words and using the wrong form of the word becomes normal. For example, in speaking form of Persian language speakers put "e" at the end of sentences as the verb "ast" (to be): "هو اخوبه" (hava khube) instead of "هوا خوب است" ( hava khub ast, the weather is good). Now, in many text messages, unfortunately, we encounter with this form as the main form of writing.

The existence of different unreliable teaching sites. The existence of different sites for teaching language that may not teach properly. Today, many non-specialists launch blogs and sites with the aim of giving information about the Persian language or just making money. These materials are usually collected from other sites and scripts, instead of using the books as reliable source, thus, there is no way to post content correctly.

As a further matter, if mistakes in written and spoken language become pervasive, the difference and gap between speaking and official writing become more common and this makes learning language more difficult for learners.

When learners start to learn the Persian language they begin with formal language, especially for writing. The language is used in internet-based applications like Facebook, Wechat, Tweeter, and so on, for chatting and communication is casual language. Therefore, there is a possibility that learners especially beginners become confused. 
The apps and media limitations. The use of stickers consequently causes the gradual reduction in lexical knowledge.

One of the components that show people's literacy is the level of their vocabulary knowledge. Some words are used less and less and they are substituted by stickers or abbreviations, thus they would be omitted from the circle of popular vocabulary and this circle becomes smaller and smaller.

The internet reinforces the accents and dialects that have more power and spoken by more people thus affect and threaten the dialects that fewer people speak. About the Persian language, although dialects or accents like Azari, Kurdish, Lori, Gilaki, and so on exist in different channels of Telegram and Instagram and some more sites and applications, they are weaker than the formal one because the formal Persian is different with them and their speakers must use the standard Persian language in order to read or search.

With the formation of cyberspace and its extraordinary admissibility, a language that used more widely in the tools of this media is becoming powerful and because it is used repeatedly, the audiences accept it with more enthusiasm.

In the meantime, users are less aware of their original language and afterward have less use of it due to their need and preference to learn and utilize the language of cyberspace. Subsequently, the official and main language of that region and country which is not parallel to the net-language would be threatened in some ways.

Moreover, the use of this cyberspace with restrictions such as time, space, volume, cost, and etc is why users try to use fewer characters to speak and write thus the appearance and language syntax is subject to change which some of them remain permanently in the language. A result of this is undesirable changing in domestic resources.

Some programs force users to write short in sections such as Twitter (140 characters). On IRC (internet relay chat) acronyms help a real-time typed conversation speed up. On mobile phones, they minimize the inconvenience of typing with tiny keys.

About the Persian language, the difference in messages' cost for Persian and English SMS is also effective in not using Persian SMS. Persian users pay more for Persian messages than English messages (out of Iran) or some phones and tablets do not support Persian fonts, in these conditions users prefer to use Finglish (writing the Persian language in English alphabets) or words that have been promoted partially in the virtual world. The Persian language and script get losses which are leading to irreversible damages.

The weakness of the responsible executive. Lack of suitable equivalents for words that enter into the communicative vocabulary in the vast volume of the internet. For these new words, the "Persian Language and Literature Academy" has created equivalents that are unusable.

There is an institute in Iran, "Persian Language and Literature Academy" which is responsible for creating new words and equivalent terms for terms that come from other languages in Persian. This institute claims that make new words but propagation of these new words need another attempt. Haddad Adel, chairman of the Persian Language and Literature Academy warned: "We do not have the executive power, and the mechanism that is foreseen for the academy is a scientific mechanism, and we cannot do all those things", he said, saying that if the mass media do not care about the Persian language, we cannot do our best. Haddad Adel addressed the mass media: 
We expect IRIB (Islamic Republic of Iran Broadcasting) and press media to be careful about the use of Persian language so that as soon as a foreign word entered is not allowed to enter the Persian language to try to find the appropriate equivalent for it. This is what we do and we expect the media to support the Academy's actions. ("The Persian Language and Alphabet Is Going to Be Destroyed in Cyberspace", 1395)

The lack of Persian resources: while, according to Mohammad Reza Rostami, the secretary of the National Cyberspace and Youth Committee, who wants to obtain knowledgeable, accurate and specialized information in every field, there is an unfortunate fact that; there are few sources in Persian which have it. Rostami adds, "This is a national identity crisis, language is considered one of the most effective tools in the development competition for any country that wants to succeed in media war and passive defense" ("Cyberspace; A Tool of Threat or an Opportunity”, 1393).

In order to preserve Farsi in the virtual world, a law was passed based on that Persian text messages sent by users are less expensive than English and Finglish SMS.

Bad effect of automatic editing programs. Automatic editing programs which correct our emails and texts are caused reducing our spelling knowledge. According to a 2010 study conducted by the English Spelling Society, more than $20 \%$ of young people who ages 18-24 reported they wouldn't feel comfortable writing an important email without a dictionary or spellcheck. With the rise of Google's auto-fill-in and our use of autocorrect, it remains to be seen how children growing up with these conveniences will be affected by them (Suico, 2013).

Although correcting software in the Persian language is not as many as in English or other languages, using this software causes laziness and is followed by low literacy. When we look up some words in the dictionary for meaning and dictation it sticks in our minds and increases our literacy.

\section{Opportunities}

Free, fast, and large access. The internet makes using videos, PowerPoint, and images possible free, fast and in a vast area to learn and teach the Persian language.

Language learners by using the internet can take advantages of many visual sources and references to learn. Visual elements are more useful for receiving all kind of information since it is so effective in learning a new language. It helps beginners have access to educational materials especially in far distant parts of the world at a lower cost and faster than the written content of the book. Language learners can download many of material for learning Persian or other languages in each part of the world that internet is accessible.

The internet helps learners to find and communicate with people who speak the Persian language thus allowing the use of Native Accents is another benefit. These days, people can meet and speak live with native Persian speaker by using Facebook, Skype, Tweeter, Instagram, Whatsapp, and other Cyberspace devices. Therefore, they can learn the formal or casual Persian language.

There are more and easier accesses to people who teach in private or language teaching institutions. There are many sites and weblogs which Persian learners can be notified about teaching advertisements by institutes or private teachers. In this way, learning the Persian language could be easier and more reasonable.

Productive and affordable travel to the country of interest and language training in the destination country could be beneficial and by using the internet, people can know about travel agencies so they can travel easier with reasonable prices.

Using online translation tools. Take advantage of simultaneous translation features like Google Translate and a variety of general and specialized online and free dictionaries to understand the concept of texts, videos, 
and music of the original language. Although this kind of translation is not as reliable as using a dictionary, in some cases and situations which is emergency Persian learner can benefit from "online translation".

\section{Creating new words.}

Not so many years ago, the word "space" had a merely geometrical meaning: the idea refers to an empty area. In scientific usage it was generally accompanied by some epithet such as "education", "isotropic" or "infinite" and the general feeling was that the concept of space was ultimately a mathematical one. Therefore, speak of "social space" would have sounded strange. (Lefebvre, 1991, p. 1)

by adding a prefix to a word like "space" resemble cyberspace which itself creates a new word. Albeit the fact that using stickers and abstracts leads to reduce the vocabulary knowledge, desirable or unsatisfactory words are being made in cyberspace. The sticker could be popular because of their easy use and their ability to show the emotion better. People from different languages can use them instead of speaking or for spending less time in writing messages and so on.

Although some of these new cyber words may not be desirable for literary and linguistic people, after years of use and staying in the vocabulary circle of individuals, they are even added to dictionaries as an undeniable part of the language like "to Google", "to tag", and so forth.

Every year, hundreds of new words and phrases that come from internet slang are added to the dictionary. Some of them are abbreviations like FOMO (Fear of Missing Out) and YOLO (You Only Live Once). Others are words that have been stretched into more parts of speech than originally intended-like when "trend" became a verb ("It's trending worldwide"). Others still have emerged as we adapt our language to new technologies; think "crowdfunding", "selfie", "cyberbullying". The secret of a new word's success is its longevity, says Fiona McPherson, Senior Editor in the New Words Group at the Oxford English Dictionary. To make it into the dictionary, the general population must use it and keep using it. A word must be in use for at least five years to be considered, McPherson says. So, love it or hate it, when words like "LOL" become common, widespread, well understood, and stick around for more than five years, they're eligible for a spot in the big book (Kolowich, 2014).

It's important to remember that a lot of internet vocabulary belongs to the category of the slang or jargon; it serves a particular purpose within an in-group, like professional slang. Hand-wringing articles appear when people from outside that in-group try to understand it, but that was never the purpose for which that vocabulary developed ("4 Ways the Internet Has Changed the English Language", 2016).

Easier and more fun. Learners have a chance to get acquainted with the culture of the target language. Students will become familiar with the attractions of that language by seeing the customs and rituals the new language has and will have more passion for learning it.

Words in different culture and society have different values and meanings. Some words are positive in the Persian language which in other languages maybe neutral, negative, or vice versa. According to Rembo "A person's social identity comes from an individual's knowledge of himself as an individual in relationship to others" ("Relationship Between Language Culture and Identity English Language Essay", 2015). The main device for communication and to make a relationship with others and with the world is language. The human cultural representation shows itself in language; words for praying, love, or hate someone or something, names of people, various kinds of greeting. These representations are different from culture to culture as a consequence from language to language. Because of that for a successful interact and communication, learning a new language is also the gate for learning the new culture. 
Another positive point of learning via cyberspace is that chat rooms in which we do not need to adhere to the rules of grammar and in some ways, make communicating possible and easy without any grammatical concerns. Typing in chat rooms most of the time consists of casual language so, learners do not need to attempt to follow grammar rules.

Finding accents and dialects. In learning a language academically or in language institutes, the language is taught, and is formal language while through the internet, learners can better understand dialects, accents, and even the street talk of a language.

Without the internet, texts that were produced in different dialects and different accents of Persian language could not be easily reproduced and made available to learners. This is not just in the Persian language; the internet provides the opportunity to preserve and protect certain regional dialects such that internet lets these dialect speakers speak and write down their unique words and phrases. Non-English speakers create a hybrid version of English by mixing it with their mother tongues so that they use English alphabet to write in other languages: Finglish (Farsi and English), Hinglish (Hindu and English), Konglish (Korean and English), and so on.

\section{Conclusion}

On the whole, the internet has accelerated the speed of language change around the world, and all the societies have certainly faced with this challenge. On the one hand, there are some foibles to learn Persian through the internet, such as the possibility for misunderstanding the words by the learners, reduction of vocabulary knowledge because of stickers, abbreviations, and short writings, making hard the learning writing for a beginner due to difference between speaking and writing forms, and so forth. On the other hand, it has several fortes like fast and easy access to native speakers, teachers and to Persian news, the ability to download Persian movies and songs, becoming familiar with culture behind the language and literature and so on.

Since the use of cyberspace and its presence in all aspects of our modern lives is inevitable, the best way to face this phenomenon is to take advantage of all its best possibilities and make its threads to opportunities. Learning and teaching in today's world have a remarkable contribution to personal development. Knowing each language is equal to better understanding its culture and vesting range of vocabulary. As a result, learning Persian should also be more active by activating applications that are able to use this language and more energetic in teaching the Persian language on the internet. We must turn, as much as possible, threats of the internet for learning the language to opportunities and more and more concentrate on the merits that this educational tool offers to us.

\section{References}

Benedikt, M. (1991). Cyberspace: First steps. Cambridge: MIT University Press.

Bourdieu, P. (1992). Language and symbolic power. Cambridge: Polity Press.

Collister, L. (2011). How is the internet affecting language? Quora. Retrieved from September 12, 2017, https://www.quora.com/How-is-the-internet-affecting-language

Crystal, D. (2001). Language and the internet. Cambridge: Cambridge University Press.

Gibson, W. (1984). Neuromancer. New York: Ace book.

Kleinman, Z. (2010). How the internet is changing language. BBC News. Retrieved from September 12, 2017, http://www.bbc.com/news/technology-10971949

Kolowich, L. (2014). The evolution of language: How internet slang changes the way we speak. HubSpot. Retrieved from September 12, 2017, https://blog.hubspot.com/marketing/how-internet-changes-language 
Lefebvre, H. (1991). The production of space. (D. Nicholson-Smith, Trans.). Oxford: Blackwell.

Mehrnews, 2371529. (1393). Cyberspace; A tool of threat or an opportunity. Mehrnews. Retrieved from September 12, 2017, http://www.mehrnews.com/news/2371529

Mehrnews, 3821543. (1395). The Persian language and alphabet is going to be destroyed in cyberspace. Mehrnews. Retrieved from September 12, 2017, http://www.mehrnews.com/news/3821543

Mitchell, W. J. (1995). City of Bits. USA: MIT University Press.

Oxford Royale Academy. (2016). 4 ways the internet has changed the English language. Oxford Royale Academy. Retrieved from September 12, 2017, https://www.oxford-royale.co.uk/articles/4-ways-internet-english-language.html

Suico, A. (2013). How the internet has changed the English language. Inbound Marketing Agents. Retrieved from September 12, 2017, http://www.inboundmarketingagents.com/inbound-marketing-agents-blog/bid/291110

Ukessays. (2015). Relationship between language culture and identity English language essay. Ukessays. Retrieved from September

12 ,

2017 , https://www.ukessays.com/essays/english-language/relationship-between-language-culture-and-identity-english-language-ess ay.php

Weareteacherfinder.com. (n.d.). How the internet and social media are changing language. Teacher Finder. Retrieved from September 12, 2017, https://www.weareteacherfinder.com/blog/internet-social-media-changing-language

Wikipedia. (n.d.). Retrieved from September 12, 2017, https://en.wikipedia.org/wiki/Persian_language 